\title{
KINETICS OF ALLOY EMISSION SPECTRA WITH DOUBLE-PULSE LASER ABLATION
}

\author{
E. S. Voropay, K. F. Ermalitskaia, \\ and F. A. Ermalitskii
}

UDC 533.9.082,533.922,533.924,535.243.2

\begin{abstract}
A spectral-kinetic recorder operating in a special time-gating mode was developed for time-shifted measurement of time-resolved spectra and emission-line intensity kinetics in atomic emission analysis. The luminescence intensity kinetics of $\mathrm{Cu}, \mathrm{Zn}, \mathrm{Sn}$, and $\mathrm{Pb}$ were recorded for two-pulse laser ablation of pure metals and multicomponent alloys. The spectral-line intensity kinetics of pure metals could be approximated with high accuracy by exponential functions. The analytical signal for ablation of multicomponent samples decayed exponentially only for interpulse laser intervals $<3 \mu \mathrm{s}$.
\end{abstract}

Keywords: laser ablation, double laser pulses, intensity kinetics, laser plasma, laser-induced breakdown spectroscopy.

Introduction. Research in molecular and atomic-emission spectrometry (AES) has historically been focused on recording spectra rather than kinetics and polarization, the other two main optical characteristics of luminescence. This circumstance is due, on one hand, to the broad distribution of very simple purely spectral instruments and, on the other, to the more complicated technology of kinetics and polarization measurements. These difficulties are exacerbated by the fundamentally nonequilibrium nature of the detected plasma discharge and preclude the use of most kinetics methods. This applies also to laser AES (LAES, laser-induced breakdown spectrometry). Intensity kinetics of several spectral lines for various elements cannot be simultaneously recorded and time-resolved spectra cannot be recorded by existing methods using PMTs to measure luminescence kinetics.

Much additional information about the physical mechanisms of the generation and existence of the nonequilibrium plasma discharge can be obtained by recording the kinetics of its behavior. Time-resolved laser-induced breakdown spectrometry (TRAES) formed a whole division of LAES [1]. TRAES was applied to a variety of topics. Most studies determined the most informative time window (interval) for recording plasma-discharge emission to exclude spectral noise in the measured signal from the quasi-continuous continuum consisting mainly of delayed and recombination luminescence $[1,2]$. Some studies visualized the behavior of the plasma at various time points after its generation $[3,4]$. Few investigations addressed classical kinetics, i.e., the kinetics of separate spectral lines and time-resolved spectra [5]. The kinetics of atomicemission lines are difficult to measure in principle and practice because, on one hand, the exciting laser pulse rate $(10 \mathrm{~Hz}$ for the most common $\mathrm{Nd}^{+}$-laser) is slow and, on the other, the recorded optical signals are weak. Kinetics recording systems have evolved from streak cameras and rotating mirrors to rapid vacuum and solid-state detectors with gating and integrating devices (including brightness amplifiers) [3-6]. The slow repetition rate of the exciting signals hinders the use of highly sensitive time-correlated photon-counting systems [7] although synchronous photon counting based on spectrographs with several detectors can be used for LAES [7]. This allows the intensity kinetics of weak spectral lines to be detected with the required uncertainty due to accumulation of multiple counts.

Experimental. A spectral-kinetic recorder operating in special time-gating mode was developed and designed at BSU for simultaneous measurement of time-resolved spectra and atomic-emission kinetics. Series of spectra with increasing time shifts of the start of recording were accumulated to measure the laser-plasma emission kinetics. The recorder operating principle was based on sequential measurements (gating sets) of spectrally separated emission curves $M$ of the studied sample by a photodetector based on a CCD array with $M$ photosensitive elements. Limitations because of its repetition rate (the standard

* To whom correspondence should be addressed.

Belarusian State University, 4 Nezavisimost' Ave., Minsk, 220030, Belarus; email: ermalitskaia@gmail.com. Translated from Zhurnal Prikladnoi Spektroskopii, Vol. 86, No. 2, pp. 283-289, March-April, 2019. Original article submitted November 12, 2018. 


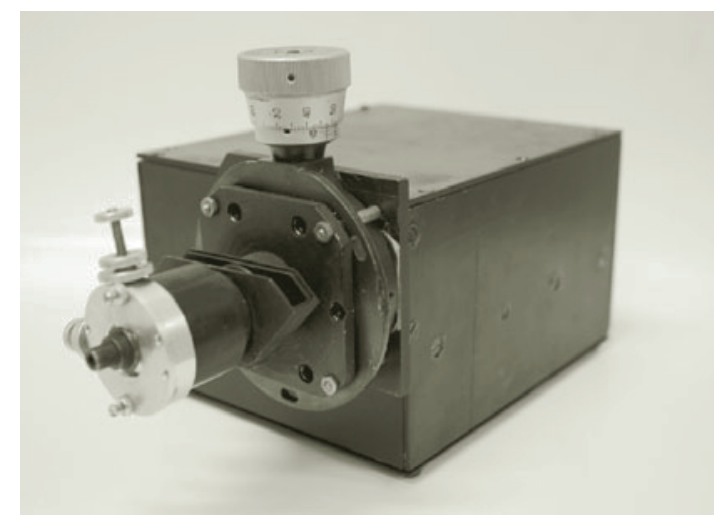

Fig. 1. Spectral-kinetic recorder operating in special time-gating mode.

length of a measurement/counting unit cycle was $\sim 10 \mathrm{~ms}$ ) were overcome by forming only one gating set for each laser pulse. Single gating sets with given time intervals were sequentially formed after the next pulses. Eventually, a time profile of $M$ plasma atomic-emission curves, each consisting of $N$ points, accumulated in the recorder after $N$ laser pulses. Spectral and kinetic curves could be summed sequentially to decrease the statistical uncertainty of the measurements. This recorder was added as a measurement channel to a commercial LSS-1 laser atomic-emission spectrometer (LOTIS TII, Belarus, Japan) [8]. It could also be used as a component of other laser, spark, or arc atomic-emission spectrometers to analyze various specimens.

The recorder was an optics-electronics system that received the radiation to be analyzed through an optical fiber, transformed the radiation into a linear spectrum using a diffraction polychromator, and detected the spectrum with a linear CCD array (Fig. 1). Radiation from the optical fiber passed through a dual-lens condenser and light filter and impinged on the entrance slit of an SDH-1 polychromator arranged in Czerny-Turner optical configuration. Light was collected at a $90^{\circ}$ angle to the discharge axis and integrated.

The instrument included the following optical and electronic elements.

1. Compact polychromator in Czerny-Turner configuration. An optical connector to attach the light pipe and a regulated optical slit that formed a linear image of radiation entering through the light pipe were installed at the polychromator entrance. The polychromator included a focusing lens, two concave mirrors, and a flat diffraction grating $(70 \times 70 \mathrm{~mm}$, 600 grooves $/ \mathrm{mm}$ ).

2. A 3648-elemental Si linear CCD array with single photosensitive elements $(8 \times 200 \mu \mathrm{m})$ and operating range $280-1060 \mathrm{~nm}$.

3. A microelectronic controller providing a special time-gating mode of CCD-array operation, processing its signals in a 12-bit ADC, and transmitting data to a computer. The gating step was programmed at intervals from $12.5 \mathrm{~ns}$ to $1000 \mu \mathrm{s}$. The gating upper limit was set by the need to avoid possible overlap of kinetic curves from neighboring ones with laser pulse length $100 \mathrm{~ms}$.

4. A USB communication channel with the computer. A suite of applied programs controlled the recorder operation. The recorder could measure emission spectra from specimens at given time intervals from the start (finish) of a laser pulse. The shape of the emission curve over time was measured for any selected part of the spectrum. The decay constants in uniand biexponential approximations were calculated.

Recorder technical specifications. Time measurement ranges 1, 2, 5, 10, 20, 50, 100, 200, 500, and $1000 \mu \mathrm{s}$; maximum rate of signal recording $100 \mathrm{~Hz}$ (determined by the working cycle length of the used CCD array); minimal time resolution $20 \mathrm{~ns}$. The total spectral recording range was $400-1060 \mathrm{~nm}$. The spectral resolution was $0.5 \mathrm{~nm}$ for the range $400-590 \mathrm{~nm}$, in which most metal lines were located. The recorder dimensions without the regulated entrance slit were $145 \times 125 \times 90 \mathrm{~mm}$. Powered from the grid $(220 \mathrm{~V} / 50 \mathrm{~Hz})$, power demand $15 \mathrm{~W}$, mass $1.4 \mathrm{~kg}$.

Results and Discussion. Analytical spectral lines of the elements must be selected considering several factors to create a quantitative analytical method for multicomponent alloys. For example, the selected line should be strong enough, not overlapped by lines of other alloy components, not a resonance line to avoid self-absorption, especially when determining the contents of elements at high concentrations. Elements often interact upon entering the erosion plasma in AES with electric and laser excitation so that relative coordinates must be used to construct calibration curves. However, the mechanism of this phenomenon is unclear. Kinetic studies of element spectral line intensities led to conclusions about the behavior 
TABLE 1. Main Characteristics of Analytical Spectral Lines of $\mathrm{Cu}, \mathrm{Zn}, \mathrm{Sn}$, and $\mathrm{Pb}$

\begin{tabular}{|c|c|c|c|c|c|c|c|}
\hline$\lambda, \mathrm{nm}$ & $A_{k i} \cdot 10^{7}, \mathrm{~s}^{-1}$ & $g_{k}$ & $E_{k}, \mathrm{~cm}^{-1}$ & $\lambda, \mathrm{nm}$ & $A_{k i} \cdot 10^{7}, \mathrm{~s}^{-1}$ & $g_{k}$ & $E_{k}, \mathrm{~cm}^{-1}$ \\
\hline \multicolumn{7}{|c|}{$\mathrm{Cu}$} & \multicolumn{3}{|c|}{$\mathrm{Pb}$} & 3 & 35,287 \\
\hline 510.554 & 0.2 & 4 & 30,783 & 405.780 & 9.0 & 5 & 45,443 \\
515.324 & 6 & 4 & 49,935 & 416.803 & 12.0 & 5 & 48,686 \\
521.820 & 7.5 & 6 & 49,942 & 520.143 & 1.4 & 3 & 39,257 \\
\hline \multicolumn{7}{|c|}{$\mathrm{Sn}$} \\
468.014 & 1.55 & 3 & 53,719 & 452.473 & 2.6 & 3 & 90,241 \\
472.215 & 4.58 & 3 & 53,719 & 533.233 & 9.9 & 4 & 89,288 \\
\hline
\end{tabular}

and propagation of the multicomponent ablation plasma and identified the optimal analytical lines for determining the concentration and thereby lowering the uncertainty of the quantitative analysis.

Common industrial alloys were studied and included special brass LS (Cu 66.37\%, Zn 29.20\%, Pb 1.85\%, Sn 0.35\%); high-tin bronze ( $\mathrm{Cu} 85.29 \%$, Sn 11.80\%, Zn 0.42\%, Pb 0.15\%); POS-40 solder ( $\mathrm{Pb} 60 \%$, Sn 40\%); and pure metals $\mathrm{Cu}, \mathrm{Zn}$, $\mathrm{Sn}$, and $\mathrm{Pb}$. Specimen surfaces were cleaned using emery paper before measurements were taken. The signal from the first pair of double pulses that vaporized a 3- $\mu \mathrm{m}$ layer of the specimen was not recorded. This excluded from consideration plasma from the surface layer containing oxides and contamination.

A two-pulse YAG:Nd laser at wavelength $1064 \mathrm{~nm}$, pulse length $15 \mathrm{~ns}$, and repetition rate $10 \mathrm{~Hz}$ was used to vaporize the specimen and excite plasma. The energy of each double pulse was $70 \mathrm{~mJ}$, interpulse interval 0 (two pulses impinge simultaneously on the surface), $1,3,5$, and $10 \mu \mathrm{s}$. The kinetics of the spectral lines were recorded $1 \mu \mathrm{s}$ after the second pulse of the double pulse to avoid recording luminescence of the leading plasma front expanding at supersonic speeds in air. Measurements were made in air at normal pressure.

The kinetics of the spectral line intensities of $\mathrm{Cu}, \mathrm{Zn}, \mathrm{Sn}$, and $\mathrm{Pb}$ were recorded in the range $400-590 \mathrm{~nm}$. Measurements for elemental analysis of $\mathrm{Cu}$ alloys were made more often in the UV range (300-490 nm). However, the goal of the work was to study processes occurring in the ablation plasma so the kinetics of the intensities of lines usually used to estimate the temperature and other thermodynamic parameters of the plasma were recorded. Table 1 presents the main characteristics of these lines (wavelength $\lambda$, spontaneous transition probability $A_{k i}$, level statistical weight $g_{k}$, and level energy $E_{k}$ ).

The results showed that the kinetics of the examined line intensities with two-pulse laser ablation of pure metals could be described by an exponential function. An interpulse interval of $1 \mu$ s gave the greatest determination coefficients of the approximation and the shortest intensity decay times (Table 2). The line intensity decay times increased to the level observed for one-pulse laser ablation if the interpulse interval was increased to $10 \mu \mathrm{s}$. However, the function deviated from exponential at the same time.

Figure 2 shows the kinetics of the $\mathrm{Zn} 481-\mathrm{nm}$ spectral line intensity with two-pulse ablation of the pure metal by double laser pulses of energy $70 \mathrm{~mJ}$ and interpulse interval 1 and $3 \mu$ s with recording starting $1 \mu \mathrm{s}$ after the second pulse in $3-\mu$ s steps.

The kinetics of analytical spectral line intensities were studied analogously for two-pulse laser ablation of multicomponent alloys (Table 3). The kinetics of the intensity changes for one-pulse ablation (zero interpulse interval) of brass, bronze, and POS-40 solder could also be described by exponential functions. However, in this instance, the determination coefficients were lower than for ablation of the pure metals. This circumstance indicated that excited atoms could react with other plasma components to affect the spectral line intensity decay rate. Nevertheless, the general features of the line intensity kinetics of the elements remained unchanged. Additional excitation of the expanding erosion cloud by the second pulse on going to two-pulse laser ablation increased the spatial heterogeneity of the plasma. In this instance, spatial heterogeneity should be understood as concentrations of elements in various parts of the plasma that are different from their contents in the starting specimen (component spatial heterogeneity). Table 3 suggests that the kinetics of the line intensities of the various components would be analogous if the ratio of element concentrations in each infinitely small plasma volume were the same as in the starting specimen. For example, exponential kinetics would be observed for two-pulse ablation of bronze with an interpulse interval of $10 \mu$ s for all components and not only low-melting $\mathrm{Pb}$ and $\mathrm{Sn}$ or the points would be 


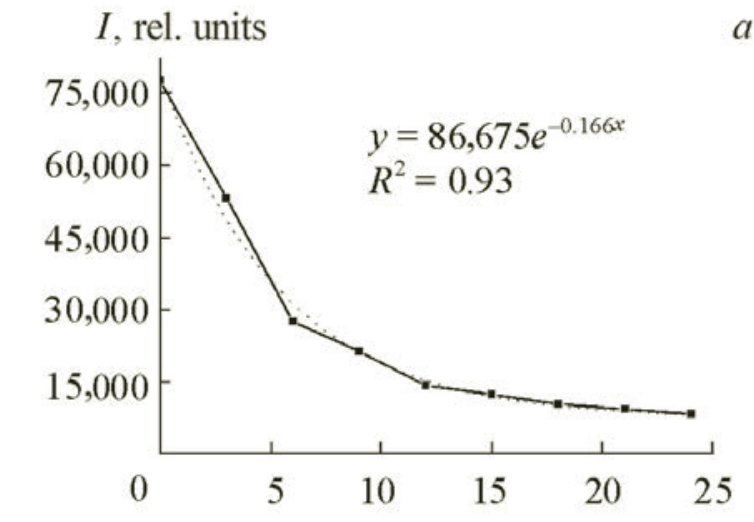

$a$

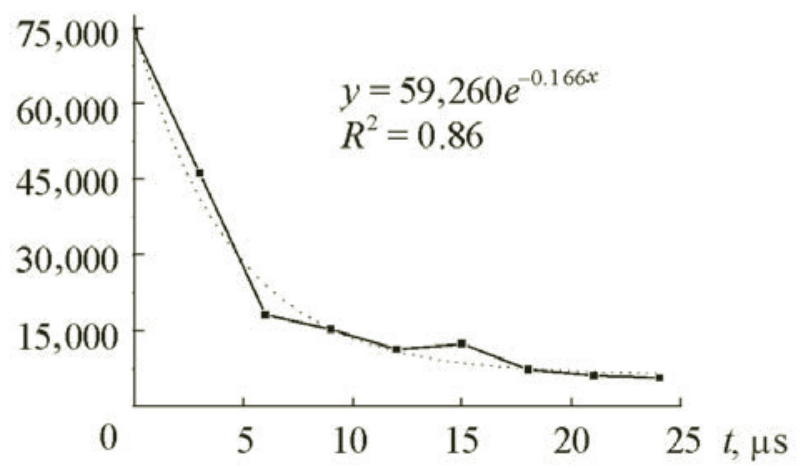

Fig. 2. Time dependence of spectral intensity of $\mathrm{Zn} 481-\mathrm{nm}$ line with double-pulse ablation of pure metal by double laser pulses of energy $70 \mathrm{~mJ}$ and interpulse interval 1 (a) and $3 \mu \mathrm{s}(\mathrm{b})$

TABLE 2. Squares of Determination Coefficients for Exponential Approximation of Metal Spectral-Line Intensity Kinetics and Decay Times

\begin{tabular}{|c|c|c|c|c|c|c|c|c|c|c|c|}
\hline \multirow{3}{*}{ Element } & \multirow{3}{*}{$\lambda, \mathrm{nm}$} & \multicolumn{10}{|c|}{ Interpulse interval, $\mu \mathrm{s}$} \\
\hline & & \multicolumn{2}{|c|}{0} & \multicolumn{2}{|c|}{1} & \multicolumn{2}{|c|}{3} & \multicolumn{2}{|c|}{5} & \multicolumn{2}{|c|}{10} \\
\hline & & $R^{2}$ & $\tau, \mu \mathrm{s}$ & $R^{2}$ & $\tau, \mu \mathrm{s}$ & $R^{2}$ & $\tau, \mu \mathrm{s}$ & $R^{2}$ & $\tau, \mu \mathrm{s}$ & $R^{2}$ & $\tau, \mu \mathrm{s}$ \\
\hline \multirow{3}{*}{$\mathrm{Cu}$} & 510.6 & 0.63 & 9.92 & 0.84 & 7.76 & 0.85 & 8.44 & 0.71 & 8.89 & 0.69 & 9.31 \\
\hline & 515.3 & 0.69 & 9.54 & 0.87 & 7.34 & 0.85 & 8.32 & 0.76 & 8.48 & 0.71 & 9.23 \\
\hline & 521.8 & 0.71 & 9.02 & 0.92 & 7.02 & 0.89 & 7.89 & 0.78 & 8.34 & 0.72 & 9.01 \\
\hline \multirow{3}{*}{$\mathrm{Zn}$} & 468.0 & 0.84 & 8.05 & 0.94 & 4.81 & 0.92 & 6.02 & 0.83 & 6.76 & 0.79 & 7.79 \\
\hline & 472.2 & 0.79 & 8.44 & 0.94 & 5.34 & 0.91 & 6.18 & 0.81 & 7.05 & 0.78 & 7.84 \\
\hline & 481.0 & 0.76 & 8.13 & 0.93 & 5.88 & 0.85 & 6.25 & 0.81 & 7.13 & 0.78 & 7.92 \\
\hline \multirow{3}{*}{$\mathrm{Pb}$} & 405.8 & 0.72 & 5.02 & 0.92 & 3.13 & 0.88 & 3.78 & 0.84 & 4.16 & 0.81 & 4.97 \\
\hline & 416.8 & 0.71 & 5.17 & 0.91 & 3.56 & 0.87 & 3.99 & 0.82 & 4.21 & 0.74 & 5.13 \\
\hline & 520.1 & 0.69 & 5.34 & 0.93 & 3.38 & 0.88 & 4.01 & 0.79 & 4.56 & 0.78 & 5.26 \\
\hline \multirow{3}{*}{$\mathrm{Sn}$} & 452.5 & 0.81 & 6.34 & 0.97 & 3.34 & 0.92 & 4.89 & 0.87 & 5.23 & 0.82 & 6.01 \\
\hline & 533.2 & 0.75 & 8.01 & 0.94 & 5.38 & 0.87 & 6.01 & 0.83 & 6.78 & 0.76 & 6.89 \\
\hline & 579.9 & 0.74 & 8.23 & 0.89 & 5.23 & 0.87 & 6.93 & 0.83 & 6.75 & 0.71 & 7.56 \\
\hline
\end{tabular}

significantly scattered for all studied spectral lines. This would indicate a considerable increase in the number of nonradiative transitions after additional excitation by the second laser pulse. However, the transition from one-pulse ablation to two-pulse for $\mathrm{Pb}$ and $\mathrm{Sn}$ produced only a slight decrease of the determination coefficient for the exponential approximation of the intensity kinetics. The simultaneous significant (up to 10 times) increase of the analytical signal made two-pulse laser ablation with interpulse intervals up to $10 \mu \mathrm{s}$ preferred for microanalysis of samples with compositions like POS-40. However, interpulse intervals $>3 \mu$ s were unfavorable for elemental analysis of $\mathrm{Cu}$ alloys because the various shapes of the intensity kinetics for the various components and especially the lack of exponential functions only for the main components and not "third" ones indicated that various elements reacted in the erosion cloud with the second laser pulse via various mechanisms. This was evident in the variable effect of nonradiative transitions on the line intensities of $\mathrm{Cu}$, $\mathrm{Zn}, \mathrm{Pb}$, and $\mathrm{Sn}$. A probable reason for this difference could be the fact that the energy of the second pulse was absorbed primarily in the erosion cloud where the element concentrations were different, e.g., mainly low-melting Pb and Sn, i.e., spatial component heterogeneity occurred. 

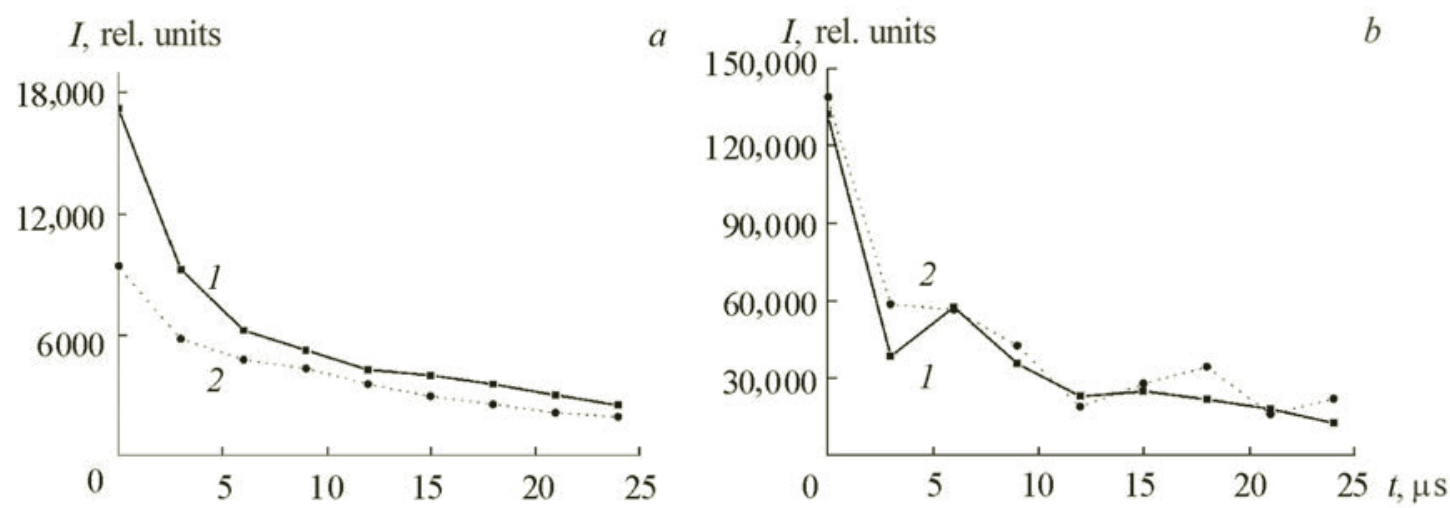

Fig. 3. Time dependence of spectral intensities of $\mathrm{Sn} 452-\mathrm{nm}$ (a) and $\mathrm{Pb} 405-\mathrm{nm}$ lines (b) for double-pulse laser ablation of solder POS-40 and interpulse interval 0 (1) and $10 \mu \mathrm{s}(2)$.

TABLE 3. Squares of Determination Coefficients for Exponential Approximation of Element Spectral Line-Intensity Kinetics and Decay Times with Double-Pulse Laser Ablation of Multicomponent Alloys

\begin{tabular}{|c|c|c|c|c|c|c|c|c|c|c|c|}
\hline \multirow{3}{*}{ Element } & \multirow{3}{*}{$\lambda, \mathrm{nm}$} & \multicolumn{10}{|c|}{ Interpulse interval, $\mu \mathrm{s}$} \\
\hline & & \multicolumn{2}{|c|}{0} & \multicolumn{2}{|c|}{1} & \multicolumn{2}{|c|}{3} & \multicolumn{2}{|c|}{5} & \multicolumn{2}{|c|}{10} \\
\hline & & $R^{2}$ & $\tau, \mu \mathrm{s}$ & $R^{2}$ & $\tau, \mu \mathrm{s}$ & $R^{2}$ & $\tau, \mu \mathrm{s}$ & $R^{2}$ & $\tau, \mu \mathrm{s}$ & $R^{2}$ & $\tau, \mu \mathrm{s}$ \\
\hline \multirow{3}{*}{$\mathrm{Cu}$ (brass) } & 510.6 & - & - & 0.61 & 10.28 & 0.61 & 10.64 & - & - & - & - \\
\hline & 515.3 & 0.59 & 11.02 & 0.68 & 9.95 & 0.56 & 10.54 & - & - & - & - \\
\hline & 521.8 & 0.62 & 10.92 & 0.72 & 9.34 & 0.70 & 9.87 & 0.61 & 10.77 & - & - \\
\hline \multirow{3}{*}{$\mathrm{Cu}$ (bronze) } & 510.6 & 0.54 & 11.08 & 0.75 & 9.02 & 0.63 & 9.56 & - & - & - & - \\
\hline & 515.3 & 0.68 & 10.56 & 0.76 & 8.94 & 0.69 & 9.37 & - & - & - & - \\
\hline & 521.8 & 0.69 & 10.12 & 0.78 & 8.61 & 0.72 & 8.92 & 0.59 & 9.96 & - & - \\
\hline \multirow{3}{*}{ Zn (brass) } & 468.0 & 0.84 & 8.05 & 0.84 & 6.87 & 0.83 & 7.82 & 0.71 & 8.76 & 0.79 & 7.79 \\
\hline & 472.2 & 0.79 & 8.44 & 0.85 & 7.39 & 0.80 & 7.98 & - & - & - & - \\
\hline & 481.0 & 0.76 & 8.13 & 0.72 & 7.98 & 0.78 & 7.65 & - & - & - & - \\
\hline \multirow{3}{*}{ Zn (bronze) } & 468.0 & 0.79 & 8.05 & 0.72 & 5.01 & 0.82 & 5.95 & 0.64 & 7.52 & - & - \\
\hline & 472.2 & 0.75 & 8.48 & 0.79 & 5.75 & 0.81 & 6.07 & 0.72 & 7.89 & - & - \\
\hline & 481.0 & 0.72 & 8.15 & 0.78 & 5.96 & 0.78 & 6.35 & - & - & - & - \\
\hline \multirow{3}{*}{$\mathrm{Pb}$ (brass) } & 405.8 & 0.72 & 4.78 & 0.90 & 2.97 & 0.78 & 3.68 & 0.62 & 4.02 & 0.81 & 4.97 \\
\hline & 416.8 & 0.71 & 5.05 & 0.87 & 3.13 & 0.75 & 3.45 & 0.61 & 4.11 & 0.74 & 5.13 \\
\hline & 520.1 & 0.69 & 5.21 & 0.85 & 3.24 & 0.72 & 3.72 & 0.68 & 4.22 & 0.78 & 5.26 \\
\hline \multirow{3}{*}{$\mathrm{Pb}$ (bronze) } & 405.8 & 0.64 & 4.53 & 0.86 & 3.05 & 0.73 & 3.78 & 0.64 & 4.01 & 0.61 & 4.24 \\
\hline & 416.8 & 0.58 & 4.24 & 0.81 & 3.21 & 0.70 & 3.99 & 0.71 & 4.09 & 0.58 & 5.07 \\
\hline & 520.1 & 0.61 & 4.83 & 0.84 & 3.17 & 0.75 & 4.01 & 0.69 & 4.21 & 0.58 & 5.12 \\
\hline \multirow{3}{*}{$\mathrm{Pb}$ (solder) } & 405.8 & 0.62 & 4.35 & 0.97 & 3.01 & 0.88 & 3.51 & 0.72 & 4.03 & 0.61 & 4.61 \\
\hline & 416.8 & 0.59 & 4.54 & 0.89 & 3.24 & 0.87 & 3.80 & 0.71 & 4.05 & 0.58 & 4.92 \\
\hline & 520.1 & 0.61 & 4.73 & 0.78 & 3.17 & 0.88 & 3.43 & 0.64 & 4.17 & 0.55 & 4.91 \\
\hline \multirow{3}{*}{ Sn (brass) } & 452.5 & 0.61 & 4.32 & 0.86 & 2.75 & 0.73 & 3.35 & 0.67 & 3.83 & 0.56 & 4.04 \\
\hline & 533.2 & 0.65 & 6.41 & 0.82 & 4.32 & 0.73 & 4.31 & 0.68 & 4.73 & 0.66 & 5.24 \\
\hline & 579.9 & 0.62 & 6.29 & 0.86 & 3.83 & 0.76 & 4.25 & 0.62 & 4.83 & 0.59 & 5.05 \\
\hline \multirow{3}{*}{ Sn (bronze) } & 452.5 & 0.78 & 6.13 & 0.93 & 3.13 & 0.88 & 4.56 & 0.72 & 5.01 & 0.64 & 5.94 \\
\hline & 533.2 & 0.63 & 7.24 & 0.91 & 4.89 & 0.81 & 5.37 & 0.70 & 6.37 & 0.62 & 6.86 \\
\hline & 579.9 & 0.61 & 7.08 & 0.78 & 4.56 & 0.72 & 5.74 & 0.71 & 6.24 & 0.67 & 6.48 \\
\hline \multirow{3}{*}{ Sn (solder) } & 452.5 & 0.81 & 6.04 & 0.92 & 4.84 & 0.87 & 4.05 & 0.87 & 5.01 & 0.72 & 5.24 \\
\hline & 533.2 & 0.75 & 7.31 & 0.90 & 5.15 & 0.84 & 5.24 & 0.83 & 6.14 & 0.65 & 6.54 \\
\hline & 579.9 & 0.74 & 7.32 & 0.91 & 5.01 & 0.81 & 6.25 & 0.83 & 6.25 & 0.71 & 6.87 \\
\hline
\end{tabular}


Figure 3 shows $\mathrm{Sb}$ 452-nm and $\mathrm{Pb}$ 405-nm line-intensity kinetics for POS-40 solder with two-pulse laser ablation and interpulse intervals of 0 and $10 \mu \mathrm{s}$.

The plasma temperature $T$ was found using spectral methods to determine the possible reasons for the deviation of the elemental line kinetics from exponential with two-pulse laser ablation. Local thermodynamic equilibrium was assumed. The Ornstein formula was used:

$$
T=\frac{0.625\left(E_{1}-E_{2}\right)}{\ln \left(g_{1} A_{1} / g_{2} A_{2}\right)-\ln \left(\lambda_{1} / \lambda_{2}\right)-\ln \left(I_{1} / I_{2}\right)} .
$$

The values were taken from Table 1. Spectra were recorded. The maximum plasma temperatures occurred for ablation of pure metals with an interpulse interval of $1 \mu \mathrm{s}: \mathrm{Cu}, 4200 \mathrm{~K} ; \mathrm{Zn}, 5600 \mathrm{~K}$; Sn, $8100 \mathrm{~K}$; and Pb, $9200 \mathrm{~K}$. The plasma temperature decreased if the interpulse interval was increased and averaged 1.5-1.8 times lower for $10 \mu$ s as compared to $1 \mu$ s. This was comparable with the temperature for one-pulse ablation. It is noteworthy that the plasma temperature reduction with increased interpulse interval for the examined elements was approximated by an exponential function $\left(R^{2}=0.91-0.93\right)$. The plasma temperatures with two-pulse laser ablation of multicomponent alloys differed for the same sample when calculated for pairs of spectral lines of different components. The difference increased with increasing interpulse interval although the temperature decreased. The temperature differences confirmed that the components were spatially heterogeneous. The lower melting elements $(\mathrm{Sn}$ and $\mathrm{Pb})$ were found primarily in the hotter plasma regions although the temperature of the vaporized cloud of multicomponent alloys averaged $500-800 \mathrm{~K}$ lower than for ablation of pure $\mathrm{Sn}$ and $\mathrm{Pb}$. The temperature kinetics with two-pulse ablation of multicomponent alloys could not be approximated by an exponential function.

Conclusions. The spectral-kinetic recorder operating in special time-gating mode could simultaneously measure time-resolved spectra and the kinetics of spectral line intensities during AES analysis. The spectral-kinetic recorder could be used in combination with an LSS-1 two-pulse laser spectrometer to estimate the kinetics of analytical spectral line intensities during ablation of pure metals and multicomponent alloys. The intensity kinetics in a multicomponent plasma did not obey an exponential function if the interpulse interval was increased over $3 \mu \mathrm{s}$. This indicated that plasma components interacted, which could additionally increase the component spatial heterogeneity in the erosion cloud, which in turn increased the uncertainty in the quantitative analysis. The element distributions in the plasma were comparatively uniform at short interpulse intervals $(\sim 1 \mu \mathrm{s})$ before the second pulse arrived and corresponded to the starting specimen. Therefore, the spectral line intensity kinetics of different components were similar. The results indicated that analytical measurements must be made at short interpulse intervals ( $1-3 \mu \mathrm{s})$ despite that fact that the maximum spectral line intensity occurred at $10 \mu$ s for two-pulse laser ablation of metals.

\section{REFERENCES}

1. P. L. Dudragne and A. Amouroux, J. Appl. Spectrosc., 52, No. 10, 1321-1327 (1998).

2. E. Grifoni, S. Legnaioli, M. Lezzerini, G. Lorenzetti, S. Pagnotta, and V. Palleschi, J. Spectrosc., 2014, Art. ID 849310 (2014).

3. W. Bauer, G. P. Perram, and T. Haugan, J. Appl. Phys., 123, 095304 (2018).

4. M. Corsi, G. Cristoforetti, M. Hildalgo, D. Iriatre, S. Legnaioli, V. Palleschi, A. Salvetti, and E. Tognoni, Appl. Spectrosc., 57, No. 6, 715-721 (2003).

5. N. Farid, S. S. Harilal, H. Ding, and A. Hassanein, J. Appl. Phys., 115, 033107 (2014).

6. G. Galbacs, N. Jedlinszki, K. Herrera, N. Omenetto, B. W. Smith, and J. D. Winefordner, Appl. Spectrosc., 64, No. 2, 161-172 (2010).

7. L. N. Vasilevich, F. A. Ermalitskii, S. V. Sukhanin, and D. V. Lipskii, Prib. Tekh. Eksp., No. 4, 163-164 (2002).

8. E. S. Voropai, I. M. Gulis, K. F. Ermalitskaya, F. A. Ermalitskii, K. N. Kaplevskii, A. E. Rad'ko, and K. A. Shevchenko, Prib. Tekh. Eksp., No. 4, 154-155 (2016). 